\title{
When is it OK to be drunk? Situational and cultural variations in the acceptability of visible intoxication in the UK and Norway
}

Eivind Grip Fjær, Willy Pedersen, Tilmann von Soest, and Paul Gray

\begin{abstract}
Background: Research on norms regulating drunken behaviour has tended to focus on differences between different countries and cultures rather than variations within them. Here, we examine whether there are: (i) situation-specific differences in the acceptability of visible intoxication among students in the UK and Norway; (ii) whether there are situation-specific and overall differences in this regard between the two countries; and finally (iii) to what degree possible differences reflect individual characteristics such as use of alcohol, perceived harm of alcohol consumption, and broader value orientation. Methods: Students at one British $(n=473)$ and one Norwegian $(n=472)$ university responded to a survey including a battery of questions assessing the acceptability of visible intoxication in different situations, such as with friends, with work colleagues, with family members, and situations where children are present. Data was also collected regarding alcohol consumption, perceived harms of alcohol consumption, and value orientation. Analyses of covariance were performed to assess patterns in the acceptability of visible intoxication across different situations, and the relative contributions of country, alcohol consumption, perceived harm of alcohol consumption and human values. Results: In both countries, visible intoxication was rated as most acceptable in situations involving friends and colleagues. Students from both countries rated visible intoxication least acceptable in situations where children are present. However, both overall, and in situations where children or family members are present, acceptability of visible intoxication scores were higher in the UK than Norway. These differences persisted after control for other variables. Conclusion: The study demonstrates large situational variation in acceptability of drunken behaviour, pointing to a fine-meshed set of norms regulating alcohol
\end{abstract}


use and drunken behaviour within the two cultures, with the UK standing out as a more alcohol-liberal culture than Norway. Such differences underline how norms regulating drunken behaviour are culturally constituted.

Key words: Drunken behaviour, Intoxication, Drunkenness, Drinking culture, Human values, Drinking norms

\section{INTRODUCTION}

Drinking occasions can give rise to seemingly chaotic and risky behaviour because they offer participants a "time out" from the demands and restrictions of everyday life (MacAndrew \& Edgerton, 1969, p. 90). Still, drunken transgressions are only acceptable "within-limits"; that is, however drunk you become, there are some limits you do not cross, some norms you do not breach (MacAndrew \& Edgerton, 1969, p. 67). Almost half a century after it was presented, this argument by MacAndrew and Edgerton remains unchallenged within alcohol research (Källmén \& Gustafson, 1998; Room, 2001), especially in studies of cross-cultural variation (e.g. Kuendig et al., 2008; Kuntsche, Rehm, \& Gmel, 2004) and studies focusing on social interaction (e.g. Abbey, 2011; Sexton, 2001). However, variations in drunken behaviour within the same cultures has to a large degree been left unexplored (Abel \& Plumridge, 2004).

Here we will narrow our focus to probably the most salient aspect of drunken behaviour: visible intoxication. Moral acceptance of intoxication is uncommon (Room, 2005, p. 149). Still, situational variation in the acceptability of visible intoxication is presumably larger than more widely condemned sexual or violent transgressions under similar circumstances. Therefore, visible intoxication can be a promising place to start when exploring intra-cultural or situational patterns in drunken behaviour. To examine the 
circumstances that may affect the acceptability of drunken behaviours, we have developed an instrument measuring the acceptability of visible intoxication in different situations, and put this to use in a survey of university students in the UK and Norway. By comparing the two countries we can separate culturally constituted patterns in the acceptability of visible intoxication from factors on the individual level.

\section{Situational norms for drunken behaviour}

Situational variation in norms regulating drinking and drunkenness may be due to differences between more established local drinking practices (Monk \& Heim, 2014, pp. 275-276;

Mäkelä, 1986). By participating in such drinking practices people may learn how to interpret and perceive the possibilities and limitations in a concrete drinking situation (Harnett, Thom, Herring, \& Kelly, 2000; Østergaard, 2009), enabling them to spontaneously behave "within limits" and judge the behaviour of others (Haidt, 2001). Therefore, people often intuitively know that different norms for drunken behaviour are sanctioned for different categories of people in the context of different drinking practices (Fjær \& Pedersen, 2015). Individuals may then seek out certain drinking practices - the contexts of situations - where temporarily excused transgressions may occur (Demant \& Törrönen, 2011). Social situations are, accordingly, an appropriate unit of analysis in the study of drunken behaviour, in addition to the study of cultural and individual differences (for an alternative approach, see Abel \& Plumridge, 2004).

Norms regulating drunken behaviour differ from those regulating the amount and frequency of drinking. The latter are often studied under the topic of "drinking norms" (e.g. Baer, Stacy, \& Larimer, 1991; Borsari \& Carey, 2003). An injunctive norm to drink (a certain amount) does not necessarily entail acceptance of visible intoxication (Fjær \& Pedersen, 2015; MacAndrew \& Edgerton, 1969, p. 17). Still, previous studies may provide some leads on 
relevant situational characteristics. Notably, drinking and drunkenness is more acceptable among friends than among colleagues, and unacceptable with children present (Caetano \& Clark, 1999). Young people may also have an ambivalent relationship to drinking with their family; parents are often those who enforce limits on young people's drinking (Wood, Read, Mitchell, \& Brand, 2004). Drinking during a work day has commonly been seen as unacceptable in both the UK and Norway, yet many employees can expect colleagues to drink socially (Ames, Duke, Moore, \& Cunradi, 2009). Alcohol is also increasingly used in the "grey area" between work and leisure, such as at work meetings, seminars and on business trips (Frone \& Trinidad, 2014), further complicating the navigation between acceptable and non-acceptable behaviour.

Focusing on visible intoxication as the most salient aspect of drunken behaviour, we should expect that if the norms regulating drunken behaviour are patterned, there should also be observable situational differences in norms regulating visible intoxication. One such pattern may be found by looking at these norms in situations where different types of people are present:

RQ1. Are there situation-specific differences in norms regulating visible intoxication, related to the presence of (i) friends, (ii) colleagues, (iii) family, and (iv) children?

\section{Differences between the UK and Norway}

In order to throw even more light on how situational variation in drunken behaviour is culturally constituted, we will compare two countries usually classified as belonging to slightly different drinking cultures - the UK and Norway. Both countries belong to the cultural north on the north-south gradient in European drinking cultures. Here, binge drinking is more common than the frequent consumption of low quantities found in, for example, 
Mediterranean countries (Kuntsche et al., 2004). In both countries, about a third of drinking occasions among adolescents lead to intoxication (Babor et al., 2010, p. 35). Still, per capita consumption in Norway is only about two thirds of that in UK (WHO, 2014, pp. 228, 246). In the adult population, the proportion of drinking occasions that lead to intoxication and the prevalence of heavy episodic drinking are higher in the UK than in Norway (Babor et al., 2010, p. 35; WHO, 2014, pp. 228, 246). In both countries, alcohol use has decreased among young people after the turn of the century (HSCIC, 2015; Pedersen \& von Soest, 2015a). Also, alcohol policy in Norway is exceptionally strict, and remains so despite shifting governments and changes in method of sale (Karlsson \& Österberg, 2007). Despite explicit intentions to reform alcohol policy to reduce drinking-related problems in the UK, recent UK government policy has tended to go in a more liberal direction (Nicholls \& Greenaway, 2015).

Although consumers in both countries share a tendency to binge, more situations may be perceived as possible drinking situations in the UK. Notably, there is a long British tradition for a popular pub culture, including varieties such as "child friendly" family pubs that offer play areas (Pratten, 2003). In contrast, bars are exclusively the domain of adults in Norway, with no drinking space equivalent to the British pub marketed to families. Although, in some Norwegian families, adults may drink alcohol when children are present, it is widely perceived as problematic (Pape, Rossow, \& Storvoll, 2015).

If intra-cultural patterns in the norms regulating visible intoxication are culturally constituted, we should expect there to be observable differences between the two countries in the situation-specific norms regulating visible intoxication, with the UK being more liberal than Norway: 
RQ2: Are there differences between the UK and Norway in the acceptability of visible intoxication, in the presence of (i) friends, (ii) colleagues, (iii) family, and (iv) children?

RQ3: Are there overall differences in the degree of acceptability of visible intoxication between the UK and in Norway?

\section{Individual characteristics and human values}

Findings reported in the drinking norms literature lead us to expect that gender and alcohol consumption levels will affect the acceptability of visible intoxication (Monk \& Heim, 2014, p. 274). Students' overestimation of the typical alcohol consumption of others is higher when the target is a man, compared to when the target is a women (Lewis \& Neighbors, 2004), and self-other discrepancy in injunctive and descriptive drinking norms is higher among women (Borsari \& Carey, 2003). Studies have also shown a positive relationship between perceived normative consumption and respondents' own consumption levels (Baer et al., 1991; Carey, Borsari, Carey, \& Maisto, 2006).

More generally, alcohol norms are also embedded into broader sets of values (Fjær \& Pedersen, 2015). Values are "trans-situational goals (...) that serve as guiding principles in the life of a person or group" (Schwartz et al., 2012, p. 664). Shalom Schwartz developed a much used circumplex of ten universal human values, where the values at the same side of the circular model are motivationally-related, or compatible, while being incompatible with those on the opposite side (Schwartz et al., 2012). Of particular interest in our context is the dimension defined by the opposing higher order values Openness to change versus Conservation. Previous studies of alcohol use indicate that the former may draw norms in a 
liberal and more explorative direction and the latter in a more restrictive one (Nordfjærn \& Brunborg, 2015; Schwartz et al., 2001, pp. 536-537). Thus, the final research question is:

RQ4: To what degree might possible differences between situational norms regarding the acceptability of visible intoxication, and possible differences in this respect between the UK and Norway, reflect people's use of alcohol, smoking, cannabis use, perceived harm of alcohol consumption, religious affiliation, and human values?

\section{METHOD}

\section{Sample and procedure}

The sample consisted of undergraduate students studying social sciences such as sociology, criminology and psychology at the Manchester Metropolitan University in the UK and the University of Oslo in Norway. Both universities are situated in cities of approximately the same sizes. An approval of the study was obtained from the Internal Review Board for Research at the Department of Psychology at the University of Oslo.

A brief survey questionnaire was distributed during lecture breaks. We did not register those who declined to participate, but observations indicate that non-participation was negligible. The oral presentation of the research project to all the students and the first page of the questionnaire presented the topic and underlined that participation was anonymous and voluntary. To ensure consistency between the English and Norwegian versions of the questionnaire, the items developed for this study in Norwegian were translated to English. The back-translation technique was used to examine the adequacy of the English version, and a few minor inconsistencies were then corrected. The translators were trained sociologists, fluent in both languages. 
In all, 472 Norwegian and 473 British students completed the questionnaire. Due to missing responses, 22 Norwegian and 12 British respondents were excluded from the analysis. The total sample included in the analysis was therefore 911 students. Consistent with the trend of increasingly unequal gender distribution in Norwegian and English higher education, $74 \%$ of respondents in Norway and $77 \%$ in the UK were women (Vincent-Lancrin, 2008).

The students in the British sample were somewhat younger (Mean $[\mathrm{M}]$ age $=20.06$, standard deviation $[S D]=1.70)$ than their Norwegian counterparts $(\mathrm{M}=21.36, \mathrm{SD}=2.17 ; \mathrm{p}<.001)$.

\section{Measures}

\section{Dependent variables}

To assess the acceptability of visible intoxication we developed a new battery of questions centring on 12 different hypothetical situations (see Appendix). This section was introduced with the following request: "We are interested in how you would consider a person your own age who has been drinking so much that he/she is visibly intoxicated (e.g. inattentive, talks indistinctly, loud-mouthed) in various situations. Please answer on a scale from 1 (not at all acceptable) to 6 (completely acceptable)." Answers were subsequently recoded to $0-5$. We asked respondents to evaluate other persons of their own age to avoid conflating the measures with related concepts, such as perceived consequences of own alcohol use (Park, 2004) or alcohol expectancies (Jones, Corbin, \& Fromme, 2001).

The characteristics of a visibly intoxicated person were drawn from descriptions of the category "obviously intoxicated" in a typology listing external signs of different levels of intoxication, which the Norwegian Directorate of Health has published to reduce the serving of alcohol to overly intoxicated people (Norwegian Directorate of Health, 2013). From the typology, we chose the most neutral characteristics to avoid negative associations, leaving out 
items such as "May appear disturbing to other customers", "Has difficulties with visual fixation", and "Butterfingered".

Then, each situation was described in a sentence, for example "At a private party, late at night, with close friends" or "In the evening, on a holiday abroad, with good friends, when their child is present". Drinking companionship included "close friends"; "colleagues"; "family and relatives"; and "with small children present". Because we take these norms to only be meaningful to participants with knowledge of specific drinking practices, some contextualization was offered by including references such as "private party", "wedding”, and "on a holiday abroad, on a public beach".

Mean scores for acceptability ratings of each of the four types of situation were computed, consisting of three items each. Internal consistency was $\alpha=.66$ for acceptability of visible intoxication while friends were present. The corresponding figures for colleagues, family, and children were, $\alpha=.62, \alpha=.78$, and $\alpha=.87$, respectively. Moreover, a measure of overall acceptability was calculated by computing the mean of all 12 items $(\alpha=.88)$.

\section{Independent variables}

We asked how often the participants had drunk alcohol during the preceding 12 months, on a 6-point scale from "never" to "more than three times a week". The questionnaire also contained a battery of questions assessing the perceived harms of alcohol on five areas: physical harm; mental health conditions; dependence; injuries; and social consequences (see: Pedersen \& von Soest, 2015b). A measure of perceived alcohol harms was calculated by taking the mean of all five alcohol-related items in this battery. We asked about religious affiliation, with "No religion", "Christianity", "Islam", or “Another religion or denomination" as options. Religious affiliation was dummy coded so that Christianity, Islam, and other religions or denominations were contrasted with no religion. Human values were measured 
with a shortened version of Schwartz's Portrait Values Questionnaire (PVQ-21) (Schwartz et al., 2001). The respondents were instructed to read 12 brief, gender-neutral descriptions of people corresponding to the six values of interest; the Openness to change dimension comprises the values Self-direction, Stimulation and Hedonism, while on the opposite pole Conservation comprises the values Security, Conformity, and Tradition. For each description, the participant was asked to rate the similarity to herself/himself on a scale from 1 ("Very much like me") to 6 ("Not like me at all"). For the Norwegian sample we used the European Social Survey’s Norwegian translation of the questionnaire.

\section{Statistical procedures}

We computed descriptive statistics for the acceptability of visible intoxication in the four different types of situations, by gender and by country. Gender and country differences of acceptability ratings were tested with analyses of variance (ANOVA). We used analyses of covariance (ANCOVA) to examine whether acceptability ratings differed according to situation, gender, and country. Moreover, we examined, by means of ANCOVA, whether these factors interacted in predicting acceptability ratings. Finally, we included several covariates to control the results for the effects of the covariates. Post-hoc tests with Bonferroni corrections were conducted.

\section{RESULTS}

Descriptive statistics for the measures for acceptability of visible intoxication in different situations according to country and gender are depicted in Table 1. In both countries, women had a tendency to rate visible intoxication as less acceptable than men, across all types of situations. However, this gender difference was not significant for the situations involving family and relatives. Visible intoxication was rated as less acceptable in Norway compared to 
the UK for situations where family and relatives and where small children were present, whereas no significant differences were observed for the other two types of situation. Significant country differences were also found for the total acceptability score. None of the Country $X$ Gender interaction effects were significant ( $p>.05$; not shown in Table 1).

To examine RQ1, ANCOVA were then conducted with acceptability ratings as the dependent variable and situation, Country, and Gender as factors (Table 2). In Model 1, age was included as a covariate, as there were significant age differences between the Norwegian and the British sample. A significant main effect of Situation on acceptability was found. Post-hoc tests showed all situations to be significantly different from each other $(p<.001)$, with the exception of the acceptability among colleagues versus among family and friends, which did not differ significantly $(p=.16)$. The analyses showed that acceptability of visible intoxication was highest when with friends $(\mathrm{M}=2.77, \mathrm{SD}=1.02)$ and lowest when children are present $(\mathrm{M}=0.90, \mathrm{SD}=1.08)$, whereas the other two acceptability ratings were in between (among colleagues: $\mathrm{M}=2.41, \mathrm{SD}=1.08$; among family and relatives: $\mathrm{M}=2.55, \mathrm{SD}$ $=1.33)$.

In relation to RQ2 and RQ3, Model 1 detected a significant country main effect, indicating that, overall, visible intoxication was more accepted in the UK than Norway. When examining interaction effects, the rather strong Situation $X$ Country interaction indicated that differences in acceptability ratings between Norway and the UK varied according to the situation. More specifically, no significant differences in acceptability ratings between Norway and the UK were observed when friends or colleagues are present $(p>.05)$. However, visible intoxication was significantly more accepted in the UK when with family members, or in situations where children are present $(p<.001)$. Other interaction effects were not significant. 
To examine RQ4 we included an increasing number of individual-level variables as covariates. The significant main effect for Gender in Model 1 reflected that women were less accepting of visible intoxication than men. In Model 2, alcohol use and evaluation of alcohol harm were included as covariates in addition to age. Not surprisingly, evaluation of alcohol harm, and particularly alcohol use, were related to acceptability of visible intoxication ( $p$ $<.001)$. However, the inclusion of the two covariates did not change the results of the main and interaction effects considerably (compared to Model 1) with the exception of the main effect for Gender, which was reduced, but remained significant $(p=.04)$.

Finally, in Model 3, religion and human values were included as additional individual covariates. This inclusion changed the effect of alcohol use, which decreased considerably when compared to Model 2. Also, the Gender effect failed to reach significance when these other individual level variables were included. The other parts of the model did not change substantially. Being a Christian and the human values of Security, Stimulation, and Hedonism were significantly related to acceptability of visible intoxication.

\section{DISCUSSION}

The results revealed considerable situation- and culture-specific variations in the acceptance of visible intoxication. With regard to social situations, visible intoxication was most acceptable when in the company of friends. Least acceptable was visible intoxication in situations where children are present. Being in the company of colleagues, and being with family and relatives, were ranked in between. Overall, students in the UK were more tolerant when it comes to the acceptability of visible intoxication than students in Norway. In line with the findings of MacAndrew and Edgerton (1969) and subsequent research (Kuntsche et al., 2004; Room, 2001), this study found large variation between countries in the normative regulation of drinking behaviour. However, the results also show that there are situation- 
specific norms - varying within the two cultures — that regulate people's drunken behaviour. In short, norms regulating drunken behaviour are not culturally homogenous.

A number of individual characteristics also played a part in this picture. Women were generally more restrictive than men, and participants' own alcohol consumption was correlated with more liberal norms. Moreover, the perceived harms of alcohol use correlated negatively with the acceptability of visible intoxication. Being Christian was also associated with more restrictive norms, compared to those with no religious affiliation. Finally, a value orientation with emphasis on the importance of Hedonism and Stimulation was associated with liberal norms, while emphasis on Security worked in the opposite direction. Despite these variations on the individual level, when they were included as independent variables, both the main effect of the country and the interaction effect between country and situation remained strong. In other words, the variations we found in the acceptability of visible intoxication between situations and between the two countries must probably be understood as culturally constituted, and not only as a consequence of different characteristics on the individual level.

It follows that the normative regulation of drunken behaviour must be understood at the individual, situational, and cultural level; on the one hand, the way people perceive and evaluate a situation reflects their previous experiences in different situations, on the other, the situations are embedded in culturally meaningful drinking practices that are generated and developed by participants. An advantage of focusing on variations in the normative regulation of drunken behaviour between different situations is that neither individuals nor cultures — neither agents nor structures - are constructed as the single source of variation in the moral regulation of drinking practices.

We recruited students studying similar subjects at a university in the UK and one in Norway, and both universities were situated in cities of approximately similar size (around 
half a million inhabitants). Thus, the samples should be well suited for comparisons. However, as previous reviews have noted regarding studies of drinking norms, the fact that these samples comprise university students, makes it difficult to draw conclusions to the general population (Monk \& Heim, 2014, p. 274). The study is further limited by the overrepresentation of women in the samples from both countries. However, due to the relatively large overall sample size, more than 100 men were included in both the Norwegian and the British sample, thereby providing sufficient statistical power to examine gender differences and reveal gender specific patterns in acceptability of visible intoxication. More importantly, this study does not reveal exactly what features of British and Norwegian culture produce the difference in the acceptability of visible intoxication. Exploring variations in the acceptability of more transgressive forms of drunken behaviour is a possible next step in the study of intra-cultural variations in the moral regulation of drunken behaviour, although variation in acceptability is likely to decrease as the perceived seriousness of the transgression increases.

\section{FUNDING}

The study was partially funded by The Research Council of Norway. 


\section{REFERENCES}

Abbey, A. (2011). Alcohol's role in sexual violence perpetration: Theoretical explanations, existing evidence and future directions. Drug and Alcohol Review, 30, 481-489.

Abel, G. M., \& Plumridge, E. W. (2004). Network 'norms' or 'styles' of 'drunken comportment'? Health Education Research, 19, 492-500.

Ames, G. M., Duke, M. R., Moore, R. S., \& Cunradi, C. B. (2009). The impact of occupational culture on drinking behavior of young adults in the U.S. Navy. Journal of Mixed Methods Research, 3, 129-150.

Babor, T. F., Caetano, R., Casswell, S., Edwards, G., Giesbrecht, N., Graham, K., . . Rossow, I. (2010). Alcohol: No ordinary commodity. Oxford: Oxford University Press.

Baer, J., Stacy, A., \& Larimer, M. (1991). Biases in the perception of drinking norms among college students. Journal of Studies on Alcohol, 52, 580-586.

Borsari, B., \& Carey, K. B. (2003). Descriptive and injunctive norms in college drinking: A meta-analytic integration. Journal of Studies on Alcohol, 64, 331-341.

Caetano, R., \& Clark, C. L. (1999). Trends in situational norms and attitudes toward drinking among whites, blacks, and hispanics: 1984-1995. Drug and Alcohol Dependence, 54, $45-56$.

Carey, K. B., Borsari, B., Carey, M. P., \& Maisto, S. A. (2006). Patterns and importance of self-other differences in college drinking norms. Psychology of Addictive Behaviors, 20, 385-393.

Demant, J., \& Törrönen, J. (2011). Changing drinking styles in Denmark and Finland. Fragmentation of male and female drinking among young adults. Substance Use \& Misuse, 46, 1244-1255.

Fjær, E. G., \& Pedersen, W. (2015). Drinking and moral order: Drunken comportment revisited. Addiction Research \& Theory, 23, 449-458. 
Frone, M. R., \& Trinidad, J. R. (2014). Perceived physical availability of alcohol at work and workplace alcohol use and impairment: Testing a structural model. Psychology of Addictive Behaviors, 28, 1271-1277.

Haidt, J. (2001). The emotional dog and its rational tail: A social intuitionist approach to moral judgment. Psychological Review, 108, 814-834.

Harnett, R., Thom, B., Herring, R., \& Kelly, M. (2000). Alcohol in transition: Towards a model of young men's drinking styles. Journal of Youth Studies, 3, 61-77.

HSCIC. (2015). Statistics on alcohol. Leeds: Health and Social Care Information Centre

Jones, B. T., Corbin, W., \& Fromme, K. (2001). A review of expectancy theory and alcohol consumption. Addiction, 96, 57-72.

Karlsson, T., \& Österberg, E. (2007). Scaling alcohol control policies across Europe. Drugs: Education, Prevention, and Policy, 14, 499-511.

Kuendig, H., Plant, M. A., Plant, M. L., Miller, P., Kuntsche, S., \& Gmel, G. (2008). Alcohol-related adverse consequences: Cross-cultural variations in attribution process among young adults. The European Journal of Public Health, 18, 386-391.

Kuntsche, E., Rehm, J., \& Gmel, G. (2004). Characteristics of binge drinkers in Europe. Social Science \& Medicine, 59, 113-127.

Källmén, H., \& Gustafson, R. (1998). Alcohol and disinhibition. European Addiction Research, 4, 150-162.

Lewis, M. A., \& Neighbors, C. (2004). Gender-specific misperceptions of college student drinking norms. Psychology of Addictive Behaviors, 18, 334-339.

MacAndrew, C., \& Edgerton, R. B. (1969). Drunken comportment: A social explanation. Chicago: Aldine.

Monk, R. L., \& Heim, D. (2014). A systematic review of the alcohol norms literature: A focus on context. Drugs: Education, Prevention and Policy, 21, 263-282. 
Mäkelä, K. (1986). Attitudes towards drinking and drunkenness in four Scandinavian countries. Annals of the New York Academy of Sciences, 472, 21-32.

Nicholls, J., \& Greenaway, J. (2015). What is the problem?: Evidence, politics and alcohol policy in England and Wales, 2010-2014. Drugs: Education, Prevention and Policy, $22,135-142$.

Nordfjærn, T., \& Brunborg, G. S. (2015). Associations between human values and alcohol consumption among Norwegians in the second half of life. Substance Use \& Misuse.

Norwegian Directorate of Health. (2013). Ansvarlig vertskap. Oslo: Norwegian Directorate of Health

Pape, H., Rossow, I., \& Storvoll, E. E. (2015). Is drinking with parents associated with highrisk drinking among adolescents? European Addiction Research, 21, 291-299.

Park, C. L. (2004). Positive and negative consequences of alcohol consumption in college students. Addictive Behaviors, 29, 311-321.

Pedersen, W., \& von Soest, T. (2015a). Adolescent alcohol use and binge drinking: An 18year trend study of prevalence and correlates. Alcohol and Alcoholism, 50, 219-225.

Pedersen, W., \& von Soest, T. (2015b). Which substance is most dangerous? Perceived harm ratings among students in urban and rural Norway. Scandinavian Journal of Public Health, 43, 385-392.

Pratten, J. D. (2003). Responding to demand. Journal of Food Products Marketing, 9, 39-52.

Room, R. (2001). Intoxication and bad behaviour: Understanding cultural differences in the link. Social Science \& Medicine, 53, 189-198.

Room, R. (2005). Stigma, social inequality and alcohol and drug use. Drug and Alcohol Review, 24, 143-155. 
Schwartz, S. H., Cieciuch, J., Vecchione, M., Davidov, E., Fischer, R., Beierlein, C., . . Konty, M. (2012). Refining the theory of basic individual values. Journal of Personality and Social Psychology, 103, 663-688.

Schwartz, S. H., Melech, G., Lehmann, A., Burgess, S., Harris, M., \& Owens, V. (2001). Extending the cross-cultural validity of the theory of basic human values with a different method of measurement. Journal of Cross-Cultural Psychology, 32, 519-542.

Sexton, R. L. (2001). Ritualized inebriation, violence, and social control in Cajun Mardi Gras. Anthropological Quarterly, 74, 28-38.

Vincent-Lancrin, S. (2008). The reversal of gender inequalities in higher education: An ongoing trend Higher education to 2030 (Vol. 1, pp. 265-298). Paris: Organisation for Economic Co-operation and Development.

WHO. (2014). Global status report on alcohol and health 2014. Geneva: World Health Organization

Wood, M. D., Read, J. P., Mitchell, R. E., \& Brand, N. H. (2004). Do parents still matter? Parent and peer influences on alcohol involvement among recent high school graduates. Psychology of Addictive Behaviors, 18, 19-30.

Østergaard, J. (2009). Learning to become an alcohol user: Adolescents taking risks and parents living with uncertainty. Addiction Research \& Theory, 17, 30-53. 
Table 1

Means (standard deviations) and analysis of variance (ANOVA) results for acceptability ratings of visible intoxications in different situations for Norwegian and British women and men.

\begin{tabular}{|c|c|c|c|c|c|c|}
\hline \multirow{2}{*}{$\mathrm{N}=911$} & \multicolumn{2}{|c|}{ Norway } & \multicolumn{2}{|c|}{ United Kingdom } & \multicolumn{2}{|c|}{ ANOVA results (F-values) } \\
\hline & Women & Men & Women & Men & Gender differences & Country differences \\
\hline \multicolumn{7}{|l|}{$\begin{array}{l}\text { Acceptability of visible } \\
\text { intoxication... }\end{array}$} \\
\hline ...among friends & $2.69(0.96)$ & $2.86(0.98)$ & $2.76(1.05)$ & $2.99(1.13)$ & $6.48 *$ & 1.44 \\
\hline ...among colleagues & $2.28(1.05)$ & $2.55(0.96)$ & $2.38(1.11)$ & $2.69(1.14)$ & $12.33 * * *$ & 2.16 \\
\hline ...among family and relatives & $2.08(1.17)$ & $2.31(1.23)$ & $2.92(1.33)$ & $2.99(1.38)$ & 2.32 & $61.07 * * *$ \\
\hline ...with small children present & $0.71(1.01)$ & $0.91(1.19)$ & $0.98(1.08)$ & $1.17(1.06)$ & $5.60 *$ & $9.90 * *$ \\
\hline Total acceptability mean score & $1.94(0.85)$ & $2.16(0.88)$ & $2.26(0.94)$ & $2.46(0.99)$ & $8.88 * *$ & $19.57 * * *$ \\
\hline
\end{tabular}

Note. ${ }^{*} p<.05, * * p<.01, * * * p<.001$. 


\section{Table 2}

Analysis of Covariance (ANCOVA) results with situation, country, and gender as factors and acceptability of visible intoxication as the dependent variable. Models 1 to 3 include increasing numbers of covariates

\begin{tabular}{|c|c|c|c|}
\hline & $\begin{array}{l}\text { Model } 1 \\
\text { F-values }\end{array}$ & $\begin{array}{l}\text { Model } 2 \\
\text { F-values }\end{array}$ & $\begin{array}{l}\text { Model } 3 \\
\text { F-values }\end{array}$ \\
\hline \multicolumn{4}{|l|}{ ANCOVA main effects } \\
\hline Situation & $25.87 * * *$ & $11.73 * * *$ & $7.68^{* * *}$ \\
\hline Country & $14.84 * * *$ & $16.31 * * *$ & $17.19^{* * *}$ \\
\hline Gender & $9.58 * *$ & $4.14 *$ & 1.63 \\
\hline \multicolumn{4}{|l|}{ ANOVA interaction effects } \\
\hline Situation $X$ Country & $29.77 * * *$ & $28.28 * * *$ & $23.75 * * *$ \\
\hline Situation $X$ Gender & 1.24 & 1.39 & 2.28 \\
\hline Country $X$ Gender & .01 & .03 & .05 \\
\hline Situation $X$ Country $X$ Gender & .77 & .81 & .61 \\
\hline \multicolumn{4}{|l|}{ Covariates } \\
\hline Age & 2.44 & 1.34 & 1.78 \\
\hline Alcohol use & & $75.38 * * *$ & $23.06^{* * *}$ \\
\hline Alcohol harm evaluation & & $8.84 * *$ & $8.48^{* *}$ \\
\hline Daily smoking & & & .46 \\
\hline Cannabis use last year & & & .10 \\
\hline \multicolumn{4}{|l|}{ Religious affiliation } \\
\hline No religion & & & (reference) \\
\hline Christian religion & & & $4.77 *$ \\
\hline Muslim religion & & & .00 \\
\hline Other religions & & & .72 \\
\hline Security & & & $8.33^{* *}$ \\
\hline Conformity & & & 2.26 \\
\hline Tradition & & & .56 \\
\hline Self-direction & & & .30 \\
\hline Stimulation & & & $6.88 * *$ \\
\hline Hedonism & & & $12.43 * * *$ \\
\hline
\end{tabular}

Note. ${ }^{*} p<.05, * * p<.01, * * * p<.001$. 


\section{Appendix}

\section{Acceptability of visible intoxication questionnaire (Likert scales excluded)}

We are interested in how you would consider a person your own age who has been drinking so much that he/she is visibly intoxicated (e.g. inattentive, talks indistinctly, loud-mouthed) in various situations. Please answer on a scale from 1 (not at all acceptable) to 6 (completely acceptable).

1. At a private party, late at night, with close friends

2. On a holiday abroad, late at night, with parents

3. On a holiday abroad, on a public beach, before noon, with close friends

4. During Freshers' Week, on campus, in the afternoon, together with other students

5. At a pub, around 4 p.m., with close friends

6. At a restaurant, in the afternoon, where there also are families with small children present

7. At a pub, on a Friday afternoon, after work, with some work colleagues

8. In the evening, on a holiday abroad, with good friends, when their child is present

9. At a Christmas party, late in the evening, with family and relatives

10. In the afternoon, at work, during a colleague's 30th birthday celebration

11. At a family wedding, late in the evening, with family and relatives

12. In the evening, together with a couple of friends, who have small children who are awake 